\title{
Una revisión de Henicotherus Brèthes, 1928 (Coleoptera, Chryso- melidae, Chrysomelinae); género endémico y áptero de Chile
}

\section{A revision of Henicotherus Brèthes, 1928 (Coleoptera, Chrysomelidae, Chrysomelinae); an endemic and apterous genus from Chile}

\section{Viviane Jerez ${ }^{1, *} \&$ Jaime Pizarro-Araya ${ }^{2}$}

${ }^{1}$ Departamento de Zoología, Facultad de Ciencias Naturales y Oceanográficas, Universidad de Concepción, Casilla 160-C, Concepción, Chile.

¿2aboratorio de Entomología Ecológica (LEULS), Departamento de Biología, Facultad de Ciencias, Universidad de La Serena, Casilla 554, La Serena, Chile.

*E-mail: vijerez@udec.cl

\section{RESUMEN}

Se redescribe el género Henicotherus Brèthes, 1928 (Coleoptera, Chrysomelidae) género endémico del norte de Chile y se designa un neotipo para H. porteri Brèthes, 1928. Además, se describe una nueva especie colectada recientemente en expediciones realizadas en Isla Chañaral (Región de Atacama) e Isla Choros (Región de Coquimbo) en el archipiélago de Los Choros. Se entregan datos biológicos y distribucionales para ambas especies, y recomendaciones en relación a la conservación de las especies de Henicotherus.

Palabras clave: Chrysomelidae, endemismo, Isla Choros, Isla Chañaral, Reserva Nacional Pingüino de Humboldt, Taxonomía.

\section{ABSTRACT}

The genus Henicotherus Brèthes 1928 and H. porteri Brèthes 1928 are redescribed and a neotype is designated. A new species is described and biological and distributional data are given for both species. On the other hand, a new species registered in expeditions carried out in Chañaral Island (Atacama Region) and Choros Island (Coquimbo Region), in the Los Choros archipelago is described. Biological and distribution data for both species and recommendations regarding the conservation of Henicotherus species are given.

Keywords: Chrysomelidae, Choros Island, Chañaral Island, endemism, Pingüino de Humboldt National Reserve, Taxonomy.

\section{INTRODUCCIÓN}

La subfamilia Chrysomelinae está representada en Chile por once géneros, de los cuales seis son monoespecíficos y endémicos: Brachyhelops Fairmaire, 1885, Henicotherus Brèthes, 1928, Pataya Bechyne, 1946, Jolivetia Bechyne, 1946, Arauconomela Bechyne \& Bechyne, 1973 y Chilenomela Daccordi, 1994. Otros tres géneros, Phaedon GuérinMéneville, 1844, representado en Chile por P. cyanopterum
Guérin-Méneville, 1844 y Plagiodera (Chevrolat, 1837), representado por $P$. erythroptera Blanchard, 1851 tienen distribución cosmopolita, mientras que Blaptea (Weise, 1915) representado en Chile por Blaptea elguetai Petipierre, 2011, tiene una distribución Neotropical que incluye Colombia y Brasil. Otro género, Strichosa Chevrolat, 1843 se distribuye mayoritariamente en Chile Central, pero también está presente en zonas preandinas del sur de Chile y Argentina. Finalmente, dos especies del género Chrysolina, C. hyperici (Forster, 
1771) y C. quadrigemina (Suffrian, 1851) fueron introducidas en 1952 desde Australia y Francia respectivamente, como controladoras de Hypericcum perforatum (hierba de San Juan) (Klein 1977). Todas las especies de Chrysomelinae chilenas, a excepción de Blaptea elguetai (Petitpierre 2011) y Arauconomela wellingtonensis (Bechyne \& Bechyne 1973, Reid 2002) son ápteras, por lo cual presentan una baja capacidad de dispersión y están asociadas preferentemente a hábitats edafo-epigeos bajo piedras u hojarasca.

Henicotherus es el único género de Chrysomelinae presente en zonas áridas y semiáridas del norte de Chile; sin embargo, la descripción original fue realizada por Brèthes (1928) en base a material recolectado por Carlos Porter en 1917, supuestamente en Curacautín, una localidad del sur de Chile. Brèthes (1928) no especificó el lugar de depósito de $H$. porteri y mucho del material tipo de las especies descritas por él se hallan perdidas; dado que no ha sido posible localizar el ejemplar que sirvió de base para la descripción del género, en este trabajo designamos un neotipo para Henicotherus porteri Brèthes, 1928 y redescribimos el género y la especie. Por otra parte, colectas realizadas durante recientes investigaciones de artrópodos epigeos en la Reserva Nacional Pingüino de Humboldt, Chile (Pizarro-Araya et al. 2012, 2014a, 2014b, 2017), nos permitieron registrar y describir una nueva especie para el género en las islas Chañaral y Choros. Adicionalmente se entregan datos distribucionales para ambas especies, información sobre características del hábitat y recomendaciones relacionadas con la conservación de ambas especies.

\section{MATERIALES Y MÉTODOS}

Los especímenes estudiados están depositados en las colecciones del Laboratorio de Entomología Ecológica, Universidad de La Serena, La Serena, Chile (LEULS, Jaime Pizarro-Araya), Museo de Zoología, Universidad de Concepción, Concepción, Chile (UCCC_MZUC, Juan Carlos Ortiz) y Museo Nacional de Historia Natural, Santiago, Chile (MNHN, Mario Elgueta). Las fotografías y medidas de los especímenes fueron tomadas con un microscopio Olympus conectado a una cámara fotográfica Moticam 5.0 y Canon EOS Rebel T5i. En las descripciones se utilizó las siguientes medidas: largo del cuerpo, largo y ancho del pronoto, largo y ancho de los élitros. Para la descripción morfológica se siguió a Daccordi (1994), Reid (2002, 2006), Chamorro-Lacayo \& Konstantinov (2004), Savini \& Joly (2006), Chamorro-Lacayo et al. (2006), de Little (2011), Klass et al. (2011), Nadein (2013), Hubler \& Klaus-Dieter (2013) y Benítez-García et al. (2017).

\section{RESULTADOS}

Familia Chrysomelidae LATreille, 1802

Subfamilia Chrysomelinae Latreille, 1802

Tribu Chrysomelini LATreille, 1802

GÉnero Henicotherus Brèthes, 1928

Diagnosis. Insectos de cuerpo corto, convexo y glabro. Machos y hembras presentan el cuerpo de color amarillo ocre y los élitros con manchas negras de distinto diámetro y forma (Figs. 1a, 2a). Cabeza: hipognata, no visible en vista dorsal. Ojos ampliamente separados, pequeños, ovalados y convexos. Labro subrectangular, margen anterior emarginado y provisto de numerosas setas cortas. Frente plana y lisa, sin surcos frontales profundos. Antenas de once segmentos, ampliamente separadas, alcanzan el primer tercio de los élitros (Fig.1c). Tórax: Pronoto sub rectangular, dos veces más ancho que largo, con una carina en todos sus bordes y sin setas tricobotriales en los ángulos anteriores y posteriores; disco con puntuación fina y esparcida; margen posterior con dos surcos longitudinales que alcanzan la línea media y ubicados en el margen externo (Figs. 1a, 2a). Cavidades coxales anteriores cerradas. Proceso prosternal corto, plano, sin carinas y con el margen apical expandido hacia el hypomeron (Fig. 1d). Élitros: convexos, sin callos humerales y coaptados en la línea de sutura; disco con líneas de puntos profundos y dispuestos en forma esparcida; epipleuras más anchas en la parte anterior, sin setas visibles lateralmente y con una línea de puntos grandes y profundos en el margen superior. Alas metatorácicas ausentes. Mesoventrito sin perforación intercoxal mesotorácica. Metaendosternito con pedúnculo corto y ancho; brazos furcales largos con tendones anteriores largos y delgados (Fig. 2g). Patas: Uñas y setas tarsales simples en todas las patas; tercer tarsómero no bilobulado. Abdomen: Cinco esternitos visibles; esternito basal dos veces más largo que los restantes, superficie fuertemente puntuada con pilosidad muy corta y esparcida (Fig. 1e).

\section{Henicotherus porteri Brèthes, 1928 \\ Fig. 1}

DiAGnOSIS: Cuerpo corto, convexo y glabro, con el tegumento liso y brillante de color amarillo ocre oscuro. Labro, clípeo y frente de color café amarillento, eventualmente la frente con una mancha café oscura; pronoto con un par de manchas café, también de presencia y extensión variable; élitros con manchas negras de distinto tamaño y distribuidas irregularmente. Esternitos, coxas y trocánteres café negruzco; fémures, tibias y tarsos amarillo ocre, al igual que las antenas. 

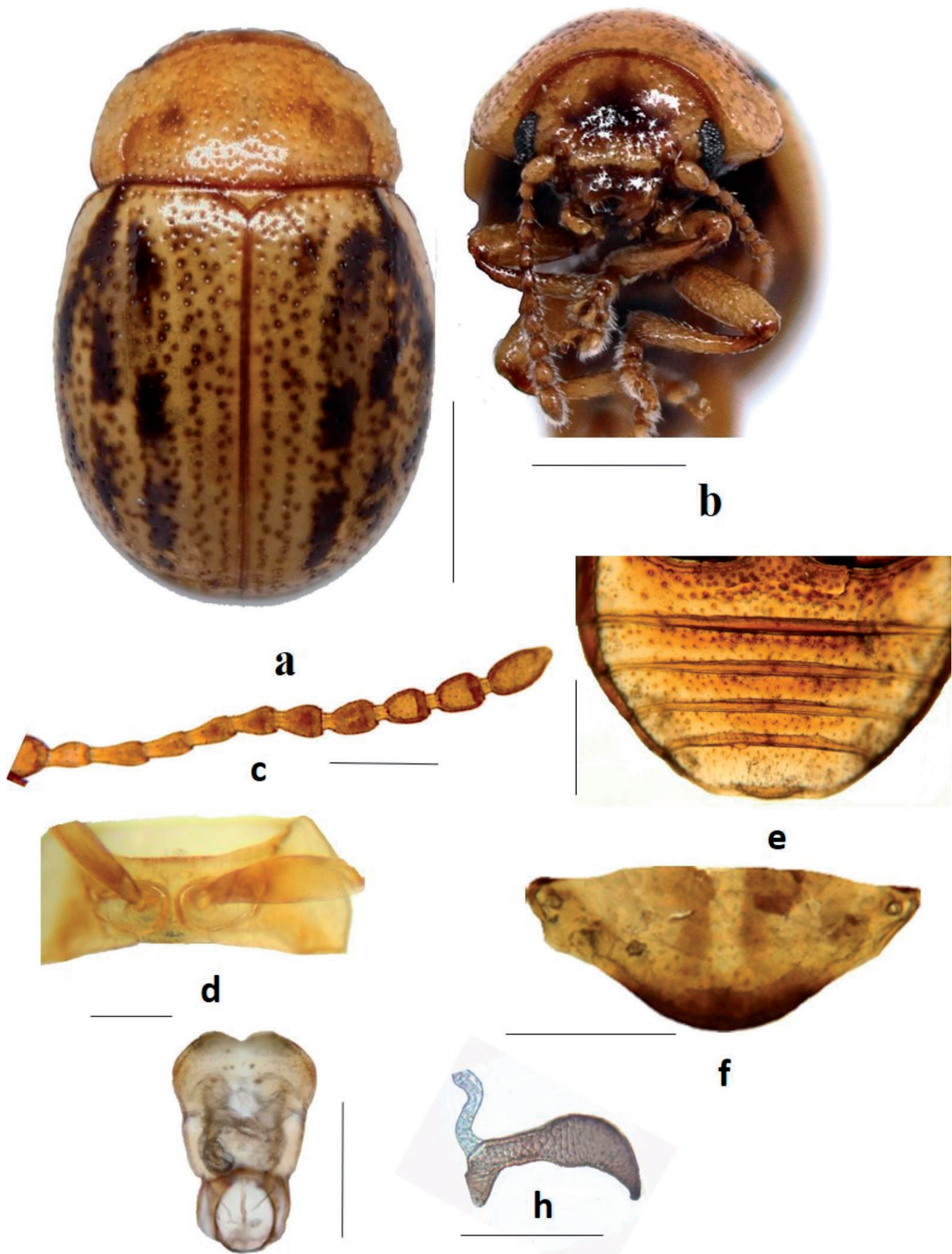

g

Figura 1. Henicotherus porteri. a) Habitus on, vista dorsal, escala: 1,0 mm; b) Cabeza vista frontal, escala: 1,0 mm; c) Antena, escala: 0,5 $\mathrm{mm}$; d) Proesterno, escala: 1,0 mm; e) Abdomen ơ, vista ventral, escala: 0,5 mm; f) Pigidio \%, vista dorsal; escala: 0,5 mm; g) Edeago, scale bar: $100 \mu \mathrm{m}$; h) Espermateca, escala: $100 \mu \mathrm{m}$. / Henicotherus porteri. a) o habitus, dorsal view, scale bar: 1,0 mm; b) Head frontal view, scale bar:1,0 mm; c) Antena, scale bar: 1,0 mm; d) Prosternum, scale bar: 0,5 mm; e) ơ abdomen, ventral view, scale bar: 0,5 mm; f) ○ Pigidium dorsal view, scale bar: 0,5 mm, g) Aedeagus, scale bar: $100 \mu \mathrm{m}$; h) Spermatheca, scale bar: $100 \mu \mathrm{m}$. 
Neotipo: ơetiquetado: Chile. Región de Coquimbo. Limarí. Fundo El Salitre. 6. XII. 2003. col. J. Pizarro-Araya (pitfall trap). El ejemplar pertenece a la colección del Museo de Zoología de la Universidad de Concepción (UCCC_MZUC). ALOTIPO: qetiquetada: Chile. Región de Coquimbo. Limarí. Fundo El Salitre. 6.XII.2003. col. J. Pizarro-Araya. Pitfall trap (UCCC_ MZUC).

Paratipos: Chile. Región de Coquimbo. Parque Nacional Fray Jorge. 25.IX.1997. Col. R. Rodríguez (UCCC_MZUC); Provincia Limarí; Fundo El Salitre, 6.XII. 2003; col. J. PizarroAraya, (10 $0^{7}, 1$ ) ), pitfall trap, (UCCC_MZUC). Quebrada Honda, 30.XI.1965. col. L.E. Peña (80",7\%), (UCCC_MZUC);

Otro Material Examinado: Región de Coquimbo, Provincia de Elqui, Choros Bajos, 20.X.1983, col. L.E. Peña (4 ejs.), Det. M. Daccordi, 1983. leg. M. Daccordi (4 ejs.) (MNHN); Cuesta Buenos Aires, subida norte, 5.II.2009, col. R. Honour (1 ej.) (MNHN); Totoralillo, Coquimbo, Huso 19, 0271718 6671870; 106 m; 30.VII. 2019, col. J. Pizarro-Araya $\left(20^{\circ}, 1\right.$ \%) (UCCC_MZUC). Provincia Limarí, Las Cardas, 27.VI.1976. col. H. Vásquez (3 ejs.) (MNHN); Parque Nacional Fray Jorge, 20.IV.1968, col. O'Brien (3 ejs.) (MNHN); Corral de Julio, km 295 (2 ejs.) (MNHN). Provincia Choapa, Camino Huentelauquén a Mincha sur, Km 4, 23-26.X.2008. col. R. Honour (2 ejs.) (MNHN).

REDESCRIPCIÓN. Modificada de Brèthes, 1928.

Medidas del Neotipo. ơ. Largo: 3,4 mm; ancho pronoto: 1,8 $\mathrm{mm}$; largo pronoto: 0,9 mm; largo élitros: $2,7 \mathrm{~mm}$; ancho élitros: 2,2 $\mathrm{mm}$. Alotipo: Largo: 3,5 $\mathrm{mm}$; largo pronoto: 1,8 $\mathrm{mm}$; ancho pronoto: $0,9 \mathrm{~mm}$; largo élitros: $2,7 \mathrm{~mm}$; ancho élitros: 2,2 mm.

\section{DESCRIPCIÓN}

Longitud: ơ: 3,1-3,5 mm ( $\mathrm{n}=5)$; 9 : 3,3-3,6 mm $(\mathrm{n}=5)$. Tanto machos como hembras presentan el cuerpo de color amarillo ocre con los élitros provistos de manchas negras de distinto diámetro y forma (Fig. 1a). La frente puede llevar una mancha de color café oscuro y está ausente algunas veces. Cuerpo ancho y convexo. Cabeza: inmersa en el protórax hasta los ojos, frente corta, plana y lisa; vertex con puntuación gruesa y esparcida, con una impresión poco profunda en la línea media. Anteclipeo subtriangular, sutura fronto clipeal fina y curvada. Ojos con pocos ommatidios (Fig. 1b). Labro corto, subrectangular, ángulos anteriores redondeados, disco con 6 poros setigeros y margen anterior emarginado. Antenas filiformes ampliamente separadas (Fig. 1b), cortas no alcanzan a la mitad de los élitros; escapo ovalado, más ancho y más largo que los restantes segmentos; segmento 2 corto y subrectangular; segmentos 3 a 6 , más largos y delgados que el segundo; segmentos 7 a 10 más anchos, cilíndricos y recubiertos de pilosidad y segmento 11 con ápice algo aguzado (Fig. 1c). Palpos maxilares con 4 segmentos, palpo distal más ancho y cónico. Mandíbulas bidentadas, prosteca membranosa. Tórax: Protórax subrectangular, convexo, dos veces más ancho que largo, márgenes laterales algo sinuados y superficie con puntuación fina y esparcida (Fig. 1a). Proceso proesternal corto, plano y expandido hacia el hypomeron (Fig. 1d). Mesoesterno no cubierto por el metaesterno; margen distal del mesoesterno y apical del metaesterno provistos de puntos setígeros grandes y profundos. Metaendosternito con pedúnculo corto y ancho; brazos furcales largos con tendones anteriores largos y delgados similar al de la nueva especie, escutelo pequeño, triangular. Élitros del ancho del tórax en la base, convexos con filas irregulares de puntos, más anchos hacia la línea media; ángulos humerales y margen apical redondeados (Fig. 1a). Epipleuras angostadas hacia el ápice, con una línea de puntos en el margen. Cada élitro lleva aproximadamente cuatro series de manchas negras dispuestas sobre las series de puntos (Fig. 1a). Abdomen: Esternitos glabros, esternito I dos veces más largo que los siguientes; en la hembra el esternito $\mathrm{V}$ presenta el margen apical redondeado y es un poco más largo que el IV; en el macho el ápice tiene un solevantamiento (Fig. 1e); pigidio sin surco en la línea media (Fig. 1f).

GENITALIA: Edeago ancho y con el ápice ampliamente curvado y emarginado; tegmen en anillo, foramen basal grande y redondeado (Fig. 1g). o: Esternito VIII y tignum con spiculum gastral corto. Espermateca falciforme, con el nodus largo y levemente curvado en la parte media; cornu con el ápice redondeado; glándula de la espermateca insertada en la base del collum; parte basal del ductus esclerificada (Fig. 1h).

HÁBITAT: H. porteri habita en terrazas costeras del matorral estepario del norte chico de Chile, bajo bosta seca y piedras. No contamos con información con respecto a los estados preimaginales.

DISTRIBUCIÓN GEOGRÁFICA: Brèthes (1928) en la descripción original señala que la especie está presente en Curacautín (Región de La Araucanía) pero hasta ahora solo ha sido registrada en la Región de Coquimbo, entre las provincias de Elqui y Limarí; por lo que consideramos como localidad errónea lo señalado por el autor (Fig. 3).

CONSERVACIÓN: La principal amenaza para esta especie sería el cambio del uso de suelo en las terrazas costeras de la región de 
Coquimbo en sectores destinados a proyectos inmobiliarios. Sin embargo, no se cuenta con datos poblacionales necesarios para su propuesta en algún estado de conservación según criterios IUCN (2012).

\section{Henicotherus francisca Jerez \& Pizarro-Araya sp. nov.}

Fig. 2

DIAGNosis. Cuerpo con el tegumento liso y brillante de color amarillo ocre oscuro. Labro, clípeo y frente de color café negruzco. Patrón de manchas en élitros: 3 manchas negras alargadas en el margen apical, 3 manchas negras mediales y un par de manchas negras alargadas en el ápice. Esternitos, coxas y trocánteres café negruzco; fémures, tibias y tarsos amarillo ocre, al igual que las antenas.

Holotipo: ơetiquetado: Chile. Región de Atacama, Provincia de Huasco, Reserva Nacional Pingüino de Humboldt, Isla Chañaral, $29^{\circ} 01^{\prime} 31.3^{\prime \prime}$ S, 71 $34^{\prime}$ '18.7" W, 35 m.s.n.m.; 1518.XI.2012. col. J. Pizarro-Araya \& F.M. Alfaro (UCCC_MZUC). Alotipo: o etiquetado: Chile. Región de Atacama, Provincia de Huasco, Reserva Nacional Pingüino de Humboldt, Isla Chañaral, $29^{\circ} 01^{\prime} 31.3^{\prime \prime}$ S, 71 $34^{\prime}$ '18.7" W, 35 m.s.n.m.; 1518.XI.2012. col. J. Pizarro-Araya \& F.M. Alfaro (UCCC_MZUC).

Paratipos: Chile. Región de Atacama. Provincia de Huasco: Isla Chañaral, $29^{\circ} 01^{\prime} 31.3^{\prime \prime}$ S, $71^{\circ} 34^{\prime}$ 18.7' W, 35 m.s.n.m., 15-18.XI.2012. col. J. Pizarro-Araya \& F.M. Alfaro, 7o'/8ㅇ

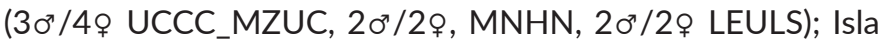
Chañaral, $29^{\circ} 01^{\prime} 44.2^{\prime \prime} \mathrm{S}, 71^{\circ} 34^{\prime} 32.3^{\prime \prime} \mathrm{W}, 40$ m.s.n.m., 15-18.XI.2012, col. J. Pizarro-Araya \& F.M. Alfaro, 18\%/20 (12\%/10 UCCC_MZUC, 6 6 /1\% LEULS); Isla Chañaral, sector E4, huso 19, 0249192-6784834, 94 msnm, 30.X.2018, col. J. Pizarro-Araya, colecta manual, $9 \% / 50^{\circ}$ (7\%/3 $0^{\circ}$ UCCC_MZUC, $20^{\prime} / 2$ o LEULS).

Otro Material Examinado. Región de Coquimbo, Reserva Nacional Pingüino de Humboldt, Isla Choros, $29^{\circ} 15^{\prime} 12,4^{\prime \prime}$

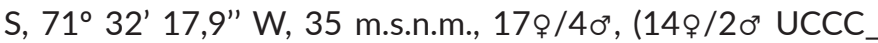
MZUC, $3 \circ / 20^{\circ}$ LEULS). col. J. Pizarro-Araya \& F.M. Alfaro.

Etımología: Esta nueva especie está dedicada a Francisca Mayora Ortiz nieta de Viviane Jerez.

Descripción. Medidas del Holotipo: ơ: Largo: $3,1 \mathrm{~mm}$; ancho pronoto:1,6 mm; largo pronoto: 0,7 mm; largo élitros: $2,5 \mathrm{~mm}$; ancho élitros: 2,2 mm. Alotipo: Largo: 4,0 mm; ancho pronoto: $1,9 \mathrm{~mm}$; largo pronoto: $1,1 \mathrm{~mm}$; largo élitros: $2,7 \mathrm{~mm}$; ancho élitros: $2,6 \mathrm{~mm}$.
Longitud. ơ: 3,1-3,7 mm ( $n=5)$. ᄋ: 4,0-4,4 mm ( $n=5)$. Cuerpo de forma ovalada y convexo, tegumento brillante y glabro. (Fig. 2a). Cabeza: Hipognata, retraída dentro del protórax; vertex con una impresión en la línea media y puntuación gruesa y esparcida; frente plana (Fig. 2b). Antenas aproximadamente la mitad del largo del cuerpo; inserciones antenales expuestas dorsalmente y ampliamente separadas; surcos frontales no fuertemente impresos. Primer segmento globoso, ancho y dos veces más largo que el segundo (Fig. 2b); segmentos 5 a 6 subrectangulares y alargados; segmentos 7 a 9 más cortos, anchos y cubiertos con pubescencia fina y densa; último segmento cilíndrico, ápice termina en punta redondeada. Ojos pequeños, con pocos ommatidios y algo emarginados (Fig. 2b). Clípeo con puntuación esparcida, sutura frontoclipeal presente. Mandíbulas bidentadas con prosteca membranosa (Fig. 2c). Labro subrectangular, emarginado; margen anterior con numerosas setas y 6 poros setigeros (Fig. 2d). Segmento apical de los palpos maxilares cónico y ápice débilmente truncado; segmento 3 corto y ancho (la mitad del largo del segmento apical); galea y lacinia apicalmente sedosa (Fig. 2e). Palpo labial con el segmento apical cónico; suturas gulares separadas. Torax. Pronoto. Largo: o: 4,1-4,4 $\mathrm{mm}$. Transverso, casi dos veces más ancho que largo con una carina lateral visible dorsalmente; disco con puntuación profunda y esparcida; ángulos anteriores y posteriores sin tricobotrios; margen posterior simple con surcos sublaterales. Escutelo pequeño, ápice triangular (Fig. 2a). Proceso proesternal plano, lados paralelos expandidos apicalmente y se unen a los lóbulos hipomerales. Proepisterno ancho, finamente carinado. Meso y metaesterno con puntuación setigera profunda que rodea las meso y metacoxas (Fig. 2f). Metaendosternito con pedúnculo corto y ancho; brazos furcales largos con tendones anteriores largos y delgados (Fig. 2g). Metacoxas transversas ampliamente separadas. Elitros: Fuertemente convexos, más anchos que el pronoto en su base y coaptados en la sutura. En la hembra son más anchos y redondeados en el ápice, en cambio en los machos son más angostos y más aguzados. Disco con puntuación profunda y esparcida dispuesta en filas longitudinales irregulares (Fig. 2a). Margen interno de la epipleura sin microsetas. Patas: bien desarrolladas y similares en tamaño; ápice de las tibias no ensanchado; todos los fémures similares en forma y tamaño. Abdomen: Esternito 1 dos veces más largo que los siguientes; todos los segmentos llevan puntuación profunda y esparcida; ultimo esternito abdominal con el ápice solevantado y en la hembra es redondeado y sin solevantamiento (carácter semejante a $\mathrm{H}$. porteri); pigidio algo aguzado en el borde marginal y sin surco en la línea media (Fig. 2h). 


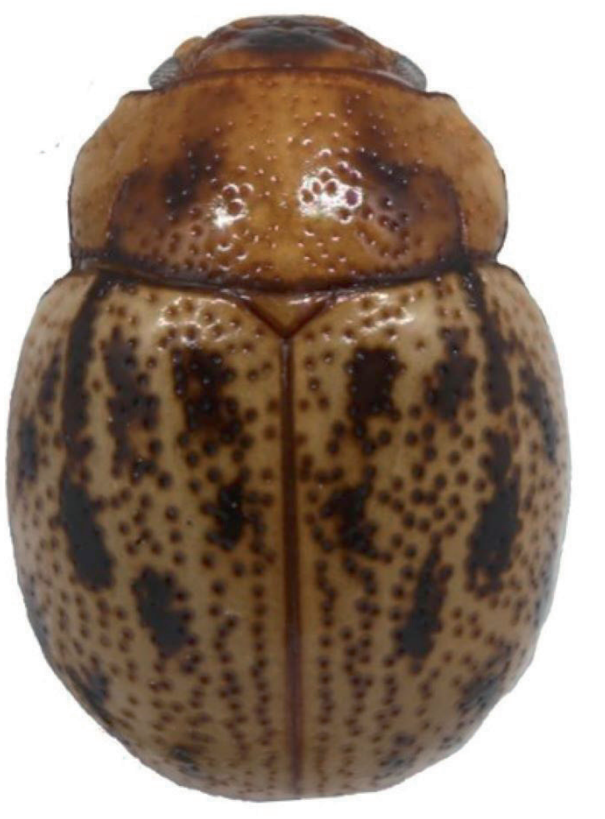

a
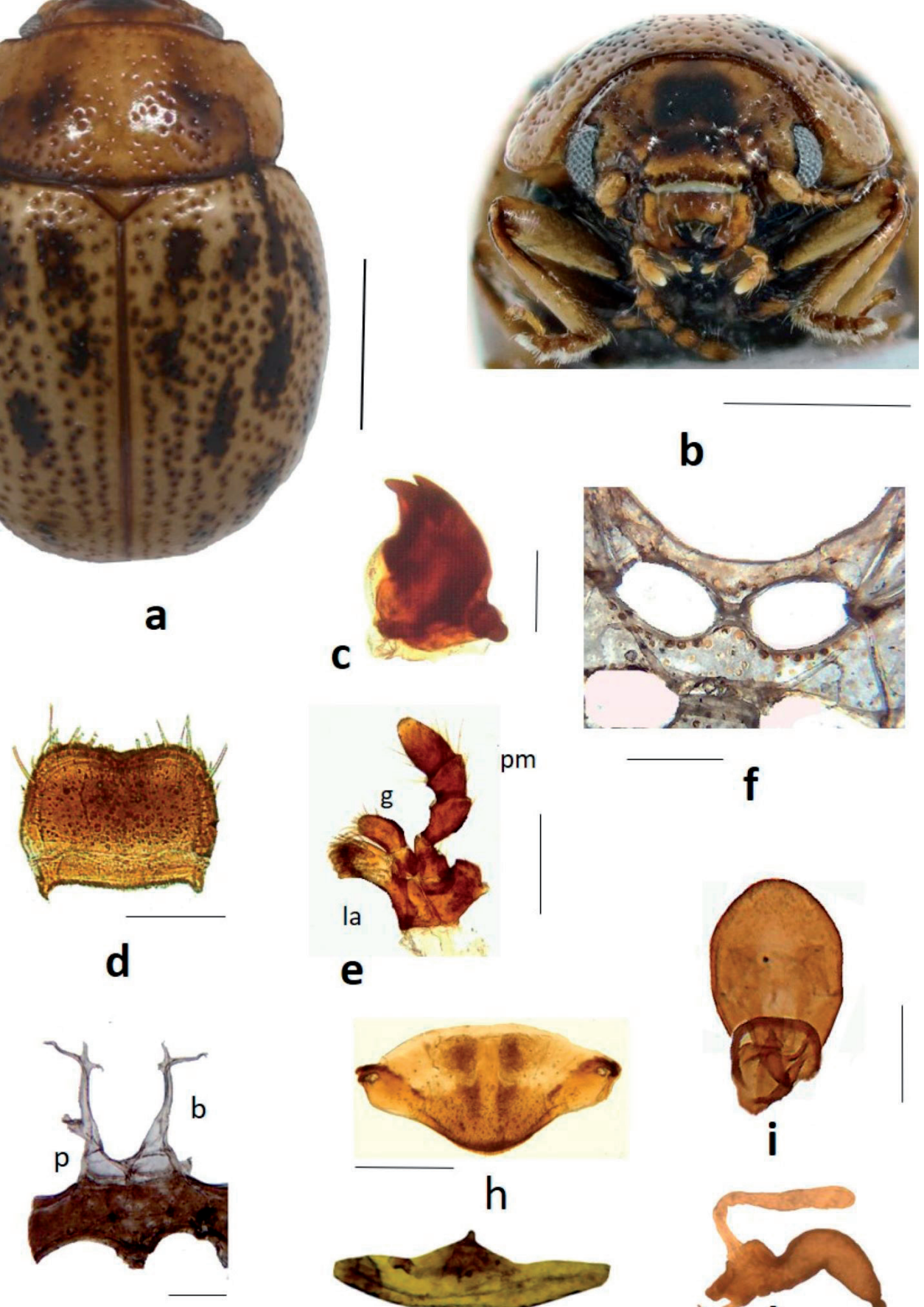

b
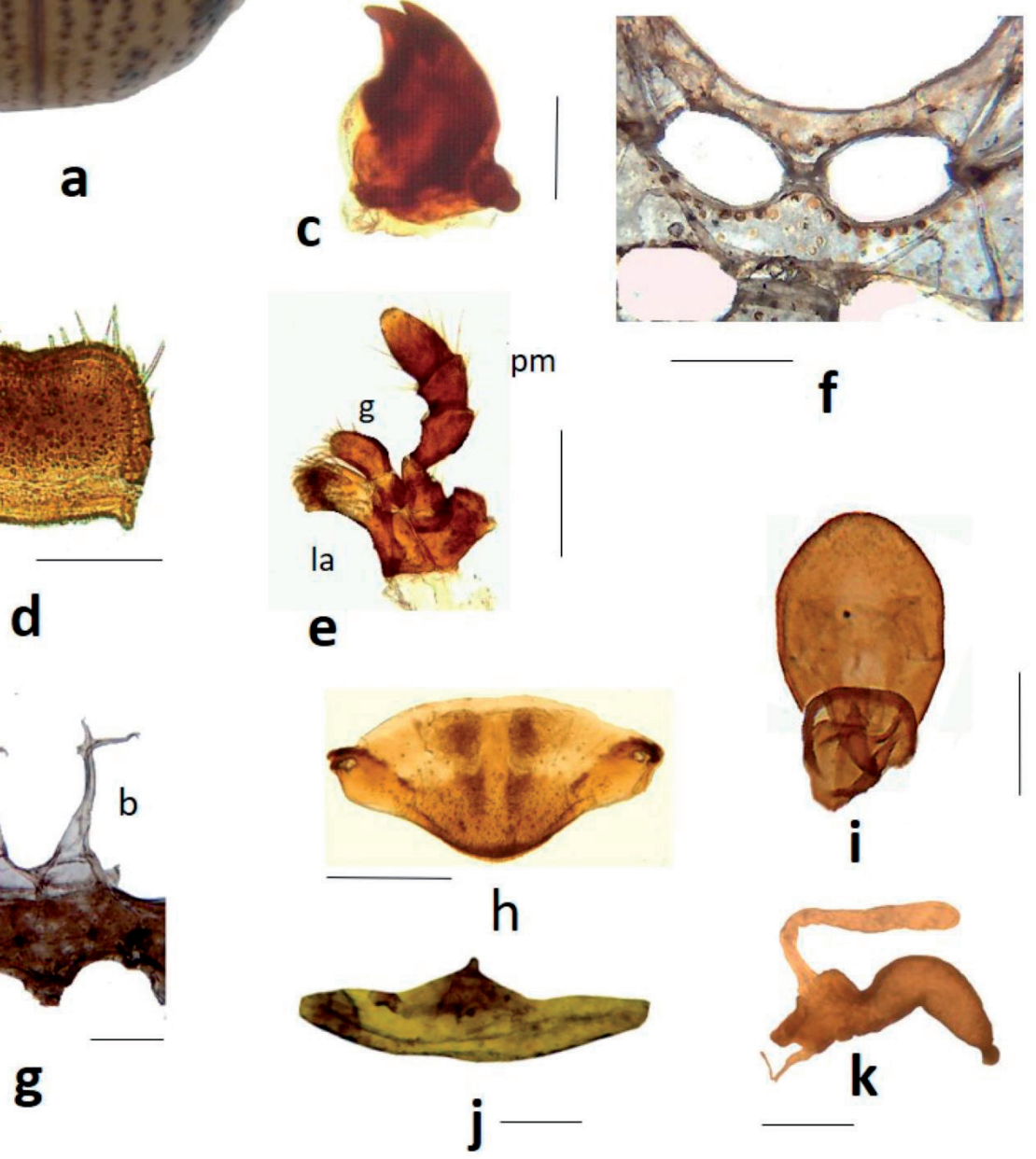

Figura 2. Henicotherus francisca sp. nov. a) Habitus vista dorsal; b) Cabeza vista frontal, escala 1,0 mm; c) Mandíbula, escala: $200 \mu \mathrm{m}$; d) Labro, escala: 200 m; e) Maxila, g: galea, la: lacinia; pm: palpo maxilar, escala: $200 \mu \mathrm{m}$; f) Meso y metaesterno, escala 1,0 mm; g) Metendosternito, b: brazo, p: pedúnculo, escala: 0,5 mm; h) Pigidio , escala: 1,0 mm; i) Edeago, escala: $100 \mu \mathrm{m}$; j) Esternito 8 y tignum, escala 0,5 mm ; k) Espermateca, escala 100 um. / Henicotherus francisca nov. sp. a) Habitus dorsal view, scale bar: 1,0 mm; b) Head dorsal

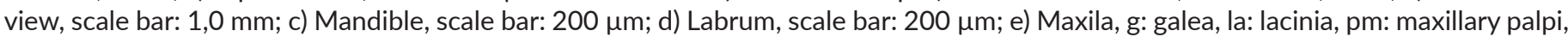
scale bar: $200 \mu \mathrm{m}$; f) Meso and metasternum, scale bar: 1,0 mm; g) Metendosternite, b: arm, p: stalk, scale bar: 0,5 mm; h) o pigidium, scale bar: 1,0 mm; i) Aedeagus, scale bar $100 \mu \mathrm{m}$; j) Sternite 8 and tignum, scale bar: 0,5 mm; k) Spermatheca, scale bar: $150 \mu \mathrm{m}$. 
Genitalia: ơ: Lóbulo medio del edeago con el ápice ancho y redondeado; tegmen ubicado en la base en forma de anillo (Fig. 2i). q: esternito 8 con spiculum gastral corto (Fig. 2j). Espermateca falciforme, con el nodus largo y fuertemente curvado en la parte media; cornu dilatado en el ápice en forma esférica; glándula de la espermateca insertada en la base del collum; parte basal del ductus corta y esclerificada (Fig. 2k).

HÁBITAT: Henicotherus francisca sp. nov. ha sido colectada en Isla Chañaral (507,3 ha) e Isla Choros (322 ha) (Reserva Nacional
Pingüino de Humboldt, Chile) (Fig. 3). Estos ecosistemas insulares corresponden a una zona costera semiárida con un clima de tipo mediterráneo influenciado por la presencia de niebla (camanchaca); las islas están rodeadas de acantilados rocosos con alturas que varían de 30 a 50 m.s.n.m. sobre el nivel del mar, con una elevación máxima de 150 m.s.n.m. (Figs. 4a, 4b). La flora de las islas está representada por diferentes formas de vida, predominantemente especies arbustivas, hierbas anuales y perennes, y cactáceas (Arancio \& Jara 2007).

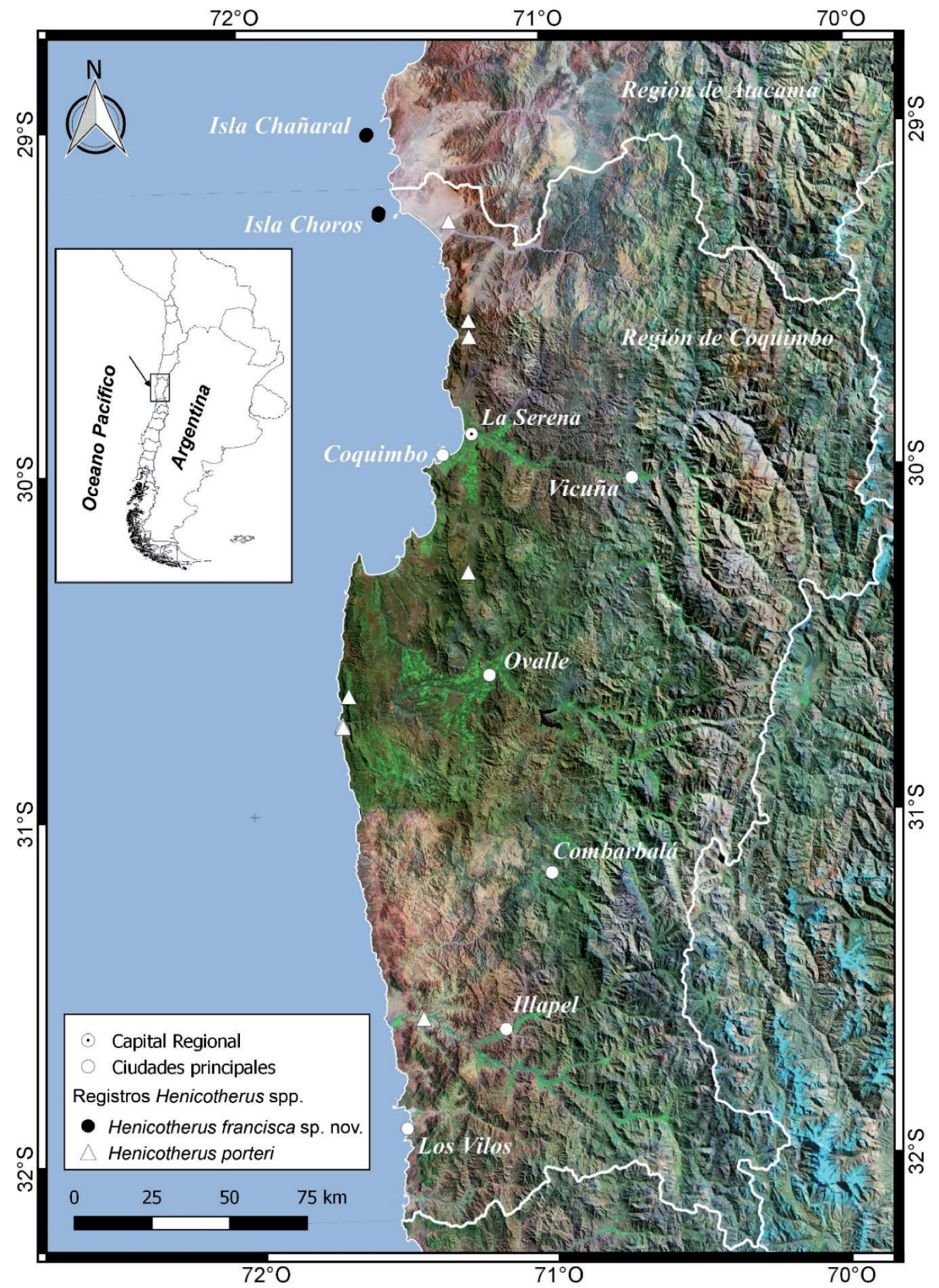

Figura 3. Registros distribucionales de Henicotherus porteri y Henicotherus francisca sp. nov. (Coleoptera, Chrysomelidae) en el desierto costero chileno. / Distribution records of Henicotherus porteri and Henicotherus francisca nov. sp. (Coleoptera, Chrysomelidae) in the Chilean coastal desert. 
Bıología: Debido a su condición áptera, Henicotherus francisca sp. nov. está asociada a sustratos rocosos, hábitat predominante en las Islas Chañaral y Choros (Fig. 3); las colectas de ejemplares vivos fueron realizadas asociadas a "piedras lajas", producto de la termoclastia -proceso de fragmentación del sustrato rocoso- (Fig. 4). Nuestras observaciones in situ y en laboratorio establecen que Henicotherus presenta hábitos fitófagos-detritívoros y edafo-epigeos, los adultos no realizan madrigueras y tienen actividad nocturna; no contamos con información con respecto a los estados preimaginales.
DistRIBUCIÓN GEOGRÁfICA: La especie Henicotherus francisca sp. nov. ha sido colectada sólo en Isla Chañaral e Isla Choros, ambas islas, junto con Isla Damas conforman la Reserva Nacional Pingüino de Humboldt, reserva localizada en el borde costero entre Chañaral de Aceituno (Región de Atacama) y Punta Choros (Región de Coquimbo) y con una superficie de 859,3 ha. Esta área pertenece al SNASPE (Sistema Nacional de Áreas Silvestres Protegidas del Estado) (CONAF 1997) (Fig. 3).
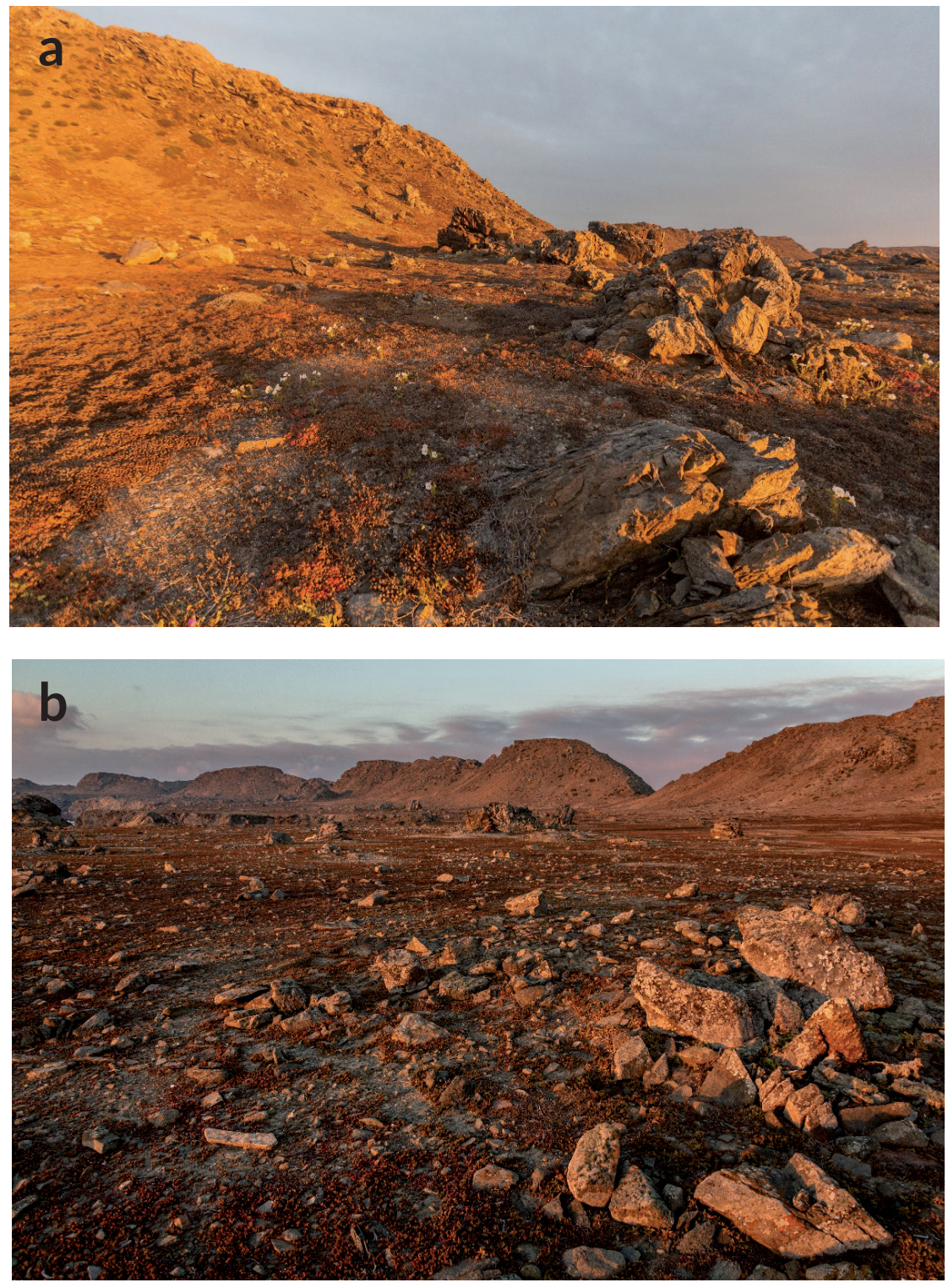

Figura 4. Hábitat de Henicotherus francisca sp. nov., a) Terrazas costeras de Isla Chañaral, exposición suroeste; b) Terrazas costeras de Isla Choros, exposición suroeste (Reserva Nacional Pingüino de Humboldt, Chile). / Habitat of Henicotherus francisca nov. sp. , a) Coastal terraces of Chañaral Island, SW exposure; b): Coastal terraces of Choros Island, SW exposure (Pingüino de Humboldt National Reserve, Chile). 
Conservación: Por lo restringido de la distribución de Henicotherus francisca sp. nov. podría ser una especie candidata para ser propuesta en los procesos de clasificación del Ministerio de Medio Ambiente (MMA), tal como lo es el coleóptero endémico de Isla Choros Gyriosomus granulipennis Pizarro-Araya \& Flores, 2004 (Coleoptera: Tenebrionidae) (Pizarro-Araya et al. 2012, 2017, Alfaro \& Pizarro-Araya et al. 2017). Consideramos importante monitorear la continuidad de esta especie, y desarrollar futuros proyectos en relación a su tamaño y dinámica poblacional, como también a las posibles amenazas y vulnerabilidades.

Comparación entre Henicotherus porterl y Henicotherus francisca SP. Nov. Henicotherus francisca sp. nov. es muy similar en coloración a $H$. porteri, pero puede ser diferenciada por su tamaño más grande y ancho, por el patrón de coloración del cuerpo que es más oscuro, y además por los siguientes caracteres. Segmento antenal 3, más largo que el 2 y pronoto con puntuación moderadamente densa en los márgenes laterales. En $\mathrm{H}$. porteri el edeago tiene el margen apical bilobulado y la espermateca presenta el nodus sin curvatura y el ápice aguzado, en cambio en $\mathrm{H}$. francisca sp. nov., el edeago tiene el ápice ancho y redondeado y la espermateca presenta el nodus con una fuerte curvatura con el ápice de forma esférica.

\section{DISCUSIÓN}

Brèthes (1871-1928) estuvo a cargo de la sección entomológica del Museo Argentino de Ciencias Naturales Bernardino Rivadavia (MACN, Buenos Aires) entre 1890 y 1928; en su descripción de Henicotherus porteri, a partir de material de crisomélidos enviados desde Chile por Porter (el autor no indica el año de envío), no entregó ninguna indicación que permita localizar el tipo en las colecciones del Museo Argentino de Ciencias Naturales Bernardino Rivadavia (MACN, Buenos Aires), Museo de La Plata (MLPA, La Plata) y Museo Miguel Lillo de Ciencias Naturales (IFML, Tucumán). Por ello consideramos necesario designar un Neotipo a partir de un ejemplar perteneciente a la colección del Museo de Zoología de la Universidad de Concepción (UCCC_MZUC).

La forma muy convexa que presentan los élitros de estos insectos y otros coleópteros que habitan en ambientes áridos y semiáridos, como ocurre con el género endémico y áptero Gyriosomus (Tenebrionidae: Nycteliini), permite la existencia de una importante cavidad subelitral que reduce la transpiración, actúa como amortiguador térmico para el flujo de calor y permite a estos coleópteros además de almacenar vapor de agua, dilatar el abdomen, almacenar alimento y guardar agua para los huevos y para la ovipostura (Jolivet 1997, Vidal et al. 2011, Tirado et al. 2018). Al respecto, los élitros muy convexos y coaptados en la sutura media de Henicotherus, constituirían una adaptación morfo-fisiológica a la deshidratación. Según Jolivet \& Verna (2005), Henicotherus tendría unas bolsas subelitrales probablemente relacionadas con la detección de agua; sin embargo, el análisis morfológico no revelo ninguna estructura presente en la parte dorsal del abdomen como ocurre en el género sudafricano Gasterantrodes (Daccordi 1994), observación que fue corroborada por Mauro Daccordi (com. pers.).

La presencia de Henicotherus francisca sp. nov. en las Islas Chañaral y Choros, se debe probablemente a un proceso de aislamiento poblacional producto de la separación de estas islas del continente, situación similar a lo señalado por Pizarro-Araya et al. (2014a) y Ceccareli et al. (2017) para escorpiones. Para estos autores, la separación existente entre las islas del norte de Chile y el continente, constituye una disyunción geográfica y sería esperable por tanto fenómenos de especiación y endemismo en ellas, como ocurre con Gyriosomus granulipennis endémico de la Isla Choros y Cyrioctea islachanaral Grismado \& Pizarro-Araya 2016 (Araneae, Zodariidae) elemento endémico de Isla Chañaral (Grismado \& Pizarro-Araya 2016).

\section{AGRADECIMIENTOS}

Agradecemos a CONAF por la ayuda en los permisos y facilidades para trabajar en la Reserva Nacional Pingüino de Humboldt (Proyectos No 18/2011, No 006/2014, N ${ }^{\circ}$ 028/2015 y 008/2017). Hacemos extensión a los colegas y amigos que participaron de las expediciones a las islas: Fermín M. Alfaro (LEULS), Alberto Castex (fundacionindomita.org), Alfredo Callejón Sánchez (fundacion-indomita. org), Tío Dogui (Punta de Choros), Patricio Ortiz (Aljavig, Chañaral de Aceituno), Cristian Rivera (Isla Chañaral, CONAF, Atacama), y Pablo Arróspide (Islas Choros y Damas, CONAF, Coquimbo). Agradecemos a Pablo Moreno por las fotografías de Henicotherus (Universidad de Concepción), a Alberto Castex por las fotografías del hábitat (FONDART N 456389 , https://www.chileasp.cl/) y a Mario Elgueta encargado de la sección de Entomología del MNHN por facilitar la revisión de material de la colección. A Luis Letelier por la asistencia en SIG (Universidad de Talca). Jaime Pizarro-Araya agradece el apoyo financiero de DIDIULS/ULS a través del proyecto PR19231210 de la Universidad de La Serena, La Serena, Chile. 


\section{REFERENCIAS}

Arancio, G., Jara, P. 2007. Flora de la Reserva Nacional Pingüino de Humbolt. Ediciones Universidad de La Serena, La Serena, Chile. 71 pp.

Alfaro, F.M., Pizarro-Araya, J. 2017. Estimación de la riqueza de coleópteros epigeos de la Reserva Nacional Pingüino de Humboldt (Regiones de Atacama y Coquimbo, Chile). Gayana 81(2): 39-51.

Bechyne, J., De Bechyne, S.P. 1973. Notas sobre algunos phytophaga de origen paleantartico. Revista Chilena de Entomología 7: 25-30.

Brèthes, J. 1928. Contribution pour la connaissance des Chrysomelides du Chili. Revista Chilena de Historia Natural 32(1): 204-220.

Benítez-García, B., López-Pérez, S., Zaragoza-Caballero, S. 2017. Synopsis of the Mexican genera of Chrysomelinae (Coleoptera: Chrysomelidae). Revista Mexicana de Biodiversidad 88(2): 335-348.

Ceccarelli, F.S., Pizarro-Araya, J., Ojanguren-Affilastro, A.A. 2017. Phylogeography and population structure of two Brachistosternus species (Scorpiones: Bothriuridae) from the Chilean coastal desert - The perils of coastal living. Biological Journal of the Linnean Society 120: 75-89.

Chamorro-Lacayo, M.L., Konstantinov, A.S. 2004. Morphology of the prothorax and procoxa in the New World Cryptocephalini (Coleoptera: Chrysomelidae: Cryptocephalinae). Zootaxa 676: 1-46.

Chamorro-Lacayo, M.L., Konstantinov, A.S., Moseyko, A.G. 2006. Comparative morphology of the female genitalia and some abdominal structures of neotropical Cryptocephalini (Coleoptera: Chrysomelidae: Cryptocephalinae). The Coleopterists Bulletin 60 (2): 113134.

CONAF. 1997. Plan de manejo Reserva Nacional Pingüino de Humboldt. CONAF Regiones de Atacama y Coquimbo, Chile.

Daccordi, M. 1994. Notes for phylogenetic study of Chrysomelinae, with descriptions of a new taxa and a list of all the known genera (Coleoptera: Chrysomelidae, Chrysomelinae). En: Furth, D.G. (Ed.) Proceedings of the Third International Symposium on the Chrysomelidae, Beijing, 1992: 60-84. Backhuys Publishers Leiden.

De Little, D.W. 2011. A revised key and notes on the Tasmanian genera of Chrysomelinae (Coleoptera: Chrysomelidae). Papers and Proceedings of the Royal Society of Tasmania 145: 17-26.

Grismado, C.J., Pizarro-Araya, J. 2016. The spider genus Cyrioctea Simon on Chañaral Island (Pingüino de Humboldt National Reserve, Atacama, Chile): description of a new species, and the male of Cyrioctea cruz Platnick (Araneae, Zodariidae). Zootaxa 4107 (2): 267-276.

Hubler, N., Klaus-Dieter, K. 2013. The morphology of the metendosternite and the anterior abdominal venter in Chrysomelinae. (Insecta: Coleoptera: Chrysomelidae). Arthropod Systematics and Phylogeny 71(1): 3-41.

Jolivet, P. 1997. Biologie des Coléopteres Chrysomelides. Société Nouvelle des Éditions Boubée. Paris. 279 pp.

Jolivet, P., Verma, K.K. 2005. Fascinating insects, some aspects to insect life. Pensoft. Sofia. Moscow. 310 pp.

Klass, K.D., Rentsch, J., Rulik, B., Hubler, N. 2011. The mesothoracic intercoxal perforation in Chrysomelinae and its evolutionary significance (Insecta: Coleoptera: Chrysomelidae). Zoologischer Anzeiger 250: 89-101.

Klein, C. 1977. Aspectos generales del control biológico e integrado de plagas en Chile. Boletín del Servicio de Defensa Contra Plagas Inspección Fitopatología 3: 121-132.

Nadein, K.S. 2013. Febraina: a new tribu of Alticini with cladistic analysis based on morphology (Coleoptera: Chrysomelidae: Galerucinae). Systematic Entomology 38(3): 491-506.

Petitpierre, E. 2011. Una nueva especie de coleóptero para la fauna chilena: Blaptea elguetai (Coleoptera: Chrysomelidae: Chrysomelinae). Boletín del Museo Nacional de Historia Natural, Chile 60: 63-66.

Pizarro-Araya, J., Vergara, O.E., Flores, G.E. 2012. Gyriosomus granulipennis (Coleoptera: Tenebrionidae) un caso extremo a conservar. Revista Chilena de Historia Natural 85(3): 345-349.

Pizarro-Araya, J., Alfaro, F.M., Flores, G.E., Letelier, L. 2017. Distribution and conservation status of Gyriosomus granulipennis Pizarro-Araya \& Flores 2004 (Coleoptera: Tenebrionidae). The Coleopterists Bulletin 71(4): 1-6.

Pizarro-Araya, J., Ojanguren-Affilastro, A.A., LópezCortés, F., Agusto, P., Briones, R., Cepeda-Pizarro, J. 2014a. Diversidad y composición estacional de la escorpiofauna (Arachnida: Scorpiones) del archipiélago Los Choros (Región de Coquimbo, Chile). Gayana 78(1): 46-56.

Pizarro-Araya, J., Alfaro, F.M., Cortes-Contreras, M., Rivera, C., Vargas-Talciani, P., Ojanguren-Affilastro, A.A. 2014b. Epigean Insects of Chañaral Island (Pinguino de Humboldt National Reserve, Atacama, Chile). Journal of the Entomological Research Society 16: 39-50.

Reid, C.A.M. 2002. A new genus of Chrysomelinae from Australia (Coleoptera: Chrysomelidae). The Coleopterist Bulletin 56(4): 589-596. 
Reid, C.A.M. 2006. A taxonomic revision of the Australian Chrysomelinae, with a key to the genera (Coleoptera: Chrysomelidae). Zootaxa 1292: 1-119.

Savini, V., Joly, L.J. 2006. Morfología de las mandíbulas de algunos géneros de Alticinae y Galerucinae (Coleoptera: Chrysomelidae). Entomotropica 21(1): 23-40.

Tirado, C., Trujillo, D., Pizarro-Araya, J., Alfaro, F.M., González, S., Carretero, M.A. 2018. Ecophysiological traits and activity patterns of coleopterans from Atacama Desert provide clues to the functional responses of small ectotherms to climate change. Journal of Arid
Environments 150: 21-27.

UICN. 2012. Categorías y Criterios de la Lista Roja de la UICN: Versión 3.1. Segunda edición. Gland, Suiza y Cambridge, Reino Unido: UICN. vi + 34pp. Originalmente publicado como IUCN Red List Categories and Criteria: Version 3.1. Second edition. Gland, Switzerland and Cambridge, UK.

Vidal, M.A., Pizarro-Araya, J., Jerez, V., Ortiz, J.C. 2011. Daily activity and thermoregulation in predatorprey interaction during the flowering desert in Chile. Journal of Arid Environments 75: 802-808.

Received: 13.11.2019

Accepted: 19.06.2020 